\section{Arbeitsgemeinschaft Medizinische Laboratoriumsdiagnostik}

\section{G. Schumann}

Hannover, Deutschland

\section{$\operatorname{Synonym(e)~AML~}$}

Definition Arbeitsgemeinschaft medizinisch-wissenschaftlicher Fachgesellschaften, die entweder ausschließlich labordiagnostisch tätig sind oder deren labordiagnostische Tätigkeit wesentlich zu ihrem Berufsbild beiträgt.

Beschreibung Die AML wurde am 21. April 1994 von 10 wissenschaftlichen Fachgesellschaften in Frankfurt gegründet. Später sind weitere Fachgesellschaften der AML beigetre- ten. Pendant auf Europäischer Ebene ist die European Confederation of Medical Laboratory (ECML), heute $>$ European Laboratory Medicine (ELM). Die AML soll fächerübergreifend der Förderung medizinischer Labordiagnostik dienen. $\mathrm{Zu}$ diesem Zweck werden regelmäßig Beratungen abgehalten und nach Bedarf Kommissionen gebildet. Die AML vertritt die Interessen labordiagnostisch tätiger wissenschaftlicher Fachgesellschaften durch Empfehlungen und Stellungnahmen zu aktuellen Problemen

Die Geschäftsstelle ist wechselnd an die Adresse des jeweiligen Vorsitzenden gebunden.

\section{Literatur}

Thomas L (1994) Arbeitsgemeinschaft Medizinische Laboratoriumsdiagnostik (AML) gegründet. Lab Med 18:342 